\title{
Perspectives on the Theory and Practice of Decision Making with the Analytic Hierarchy Process
}

\author{
Thomas L. Saaty \\ University of Pittsburgh
}

\section{The Welcome}

I want to heartily welcome all of you to this third International Symposium on the Analytic Hierarchy Process (ISAHP) held in Washington, D.C. I want to express my deep appreciation to all the attendees for coming to this meeting. On behalf of the audience I want to express our gratitude to our colleague and friend Professor Ernest Forman and his associates, Pedro Sanchez and Refik Soyer, at the George Washington University for their hard work to make this meeting with its publication materials, housing and banquets all, possible. We also want to thank the George Washington University for its hospitality to sponsor and house this three day conference on its campus. Although we do not have an AHP society, our meetings every three years keep us in touch and apprised of progress in the field. Most important they allow us to meet and exchange ideas with new colleagues from all over the world.

The AHP is spreading in academic, government and business practices. Here are some countries where there are people working with the AHP: Australia, Brazil, Bulgaria, Canada, Chile, China, Czechoslovakia, Finland, France, Germany, Holland, Hong Kong, Hungary, India, Indonesia, Israel, Italy, Japan, Kuwait, Malaysia, Mexico, Morroco, Netherlands, Portugal, Singapore, South Africa, Spain, Sweden, Taiwan, Thailand, Turkey, United Kingdom, U.S.A., and the Ukraine. One of the most cheering aspects of this symposium on the AHP is that it draws people like us together to share ideas, exchange views, exhibit applications and learn from one another. We would like to ask people from different countries to identify a contact person who would then leave his name and address at the desk so a list can be made and distributed to the attendees. These contact people would be sent the Expert Choice News containing new ideas from everybody else to distribute. More generally we can all subscribe to this biannual newsletter by paying $\$ 5.00$. Please include telephone, fax and e-mail numbers and address. It would be nice if users from different countries attending this meeting were to give the registration desk a paragraph of the uses of the AHP in their country so we can use them to represent our varied interests.

We also would like everyone here to give us, in addition to their full address, an indication in writing of their special area of interest regarding the AHP so we can begin to develop a worldwide network for AHP communication to share information.

\section{Glimpses}

Give or take; there are six billion decision makers in this world. They all make several conscious and a myriad of unconscious decisions every day. Scientific decision making is needed more and more because of increasing population and crowding, because of the complexity of our world and a rising standard of living, and because of the widespread use of technology and the desire of every country to develop economically, improve education, health, and control crime. A science of decision making is also needed because every business wants to attain its goals, increase its profits and improve its survivability in a very competitive 
environment. The call for help has become overt and frequent. If you look and listen and have not already become involved, you will find the need for our paradigm of thinking abundantly around you.

Our world has become one unit divided into some two hundred geographic countries. These countries must constantly make decisions about economic progress, technologies to develop or buy and whom to trade with. Decision making has become the invariant of all human undertakings. Intellectually, it is the funnel of information and knowledge gathered from experience and from simply noting how things unfold in the environment around us. The means by which we communicate our theories to users must develop, diversify and integrate to reach all the inhabitants of this planet.

Decision making is the science of transforming and relating data about the world to our value system so we can take the necessary actions to fulfill our needs, and aspirations. You and I are fortunate to be working and thinking in a theory that is at the cutting edge of science and value. When I say decision making I also mean conflict resolution, and when I say science, I also include the medical and health fields, genetics and human engineering.

If we were to scan varied fields of knowledge for a challenging intellectual occupation of practical consequence for the future, we could not think of anything more exciting with more noticeable results than decision making. As educators, researchers and users, we are interested in new and stimulating ideas. Decision making, planning and conflict resolution based on negotiation and compromise are flying their banners in our time. We are still very primitive at how to stop people from being killed and lack a compelling ethic to talk and not to fight. With the spread of democracy and freedom people want to express their views and these views must be reconciled in a convincing and persuasive way. We who work in decision making have an exciting challenge facing us. It is to advance decision making to a point where everyone can do it naturally and with ease and satisfaction like driving a car or owning a home in which one feeis comfortable and in command. Our ideas and their elaborations should also be useful to the experienced, knowledgeable and demanding in our midst who will be asking us about the more complex social decisions that they face.

Any theory of decision making needs to anticipate and accommodate human nature as we see it unfold. We should not make decision making so rigid that every person must spend years specializing in its intricate manipulations. It must be simple and natural - an outgrowth of our common sense, but adequately robust to be able to tumble with situations as they arise. The challenge of decision making is in fact an opportunity for people to grow and to exercise their creativity. As long as there are people, there will be the need for decision making and this need will foster intellectual growth.

I expect that students working in our field will be able to find jobs at the World Bank, the International Monetary Fund, the United Nations, energy agencies, nuclear energy facilities, in education, in health, in international business strategy, and generally in societal and global concerns where they could moderate and facilitate decision making in large organizations. I anticipate that universities will provide more business and engineering courses in planning and decision making as part of the required education curriculum. Research connecting psychology, sociology, political science measurement with decision making will increase and there will be less emphasis on statistical analysis as the basic modelling approach.

Another area in addition to decision making that needs our attention is performing measurement. It is of great concern that our old scales and measures are not adequate to describe our world today. Even our GNP figures are suspect. The figures our governments use 
derive from industrial manufacturing concerns, and are not very useful as measures in the information based society we are becoming. We suspect many economies from that of the United States to that of China are much more productive than these out-of-date measures show. New ways are needed to perform evaluations. Peter F. Drucker addressed this question in his article, "We Need to Measure, not Count," in the Wall Street Journal of April 13, 1993. We quote:

"Quantification has been the rage in business and economics these past 50 years. Accountants have proliferated as fast as lawyers. Yet we do not have the measurements we need. Neither our concepts nor our tools are adequate for the control of operations, or for managerial control. And, so far, there are neither the concepts nor the tools for business control - i.e., for economic decision making. In the past few years, however, we have become increasingly aware of the need for such measurements... It may take many years, decades perhaps, until we have the measurements we need in all these areas. But at least we know now that we need new measurements and what they have to be. Slowly, and still groping, we are moving from counting to measuring."

Another well-known philosopher that was intensely concerned with questions of value was Nietzsche. His masterpiece, Thus Spoke Zarathustra, revolves around evaluative concerns as do most of his later works. He says, "All the scientists have to from now on prepare the way for..the future task of philosophers. This task is the solution of the problem of value, the determination of the order of rank among values."

Values and knowledge are inevitably linked in and through actions. All action signifies an ethic, serves or disserves certain values. The very definition of "true" knowledge reposes in the final analysis upon an ethical [value-laden] postulate.

…...... There are nearly a half a dozen Multicriteria Decision Making Methods: the Analytic Hierarchy Process (AHP), Utility Theory, Bayesian Analysis, Outranking methods involving concordance recently extended to cardinal preference and Optimization and dominance methods. During this symposium there will be a talk by my colleague, Luis Vargas, summarizing these methods.

\section{The AHP}

More than twenty years have passed since the AHP's first major application to the Sudan Transport Project in 1973. Over a thousand papers, books, reports and dissertations have been written on the AHP. A sizeable bibliography is included in my new book, Fundamentals of the Analytic Hierarchy Process given to the participants in this meeting. An updated bibliography is available on disk for copying.

Fundamentally, the AHP differs from all other multicriteria methods in two ways. First it uses hierarchic structures to make explicit and transparent all the elements involved in a decision. The AHP also makes explicit the implicit meaning one assigns to a utility function. Criteria are evaluated according to importance, not by examining the utilities of the alternatives but by relating them to higher goals in terms of which these utilities would have to be determined. The result is that answering the question in the AHP becomes much simpler in terms of importance, preference or likelihood. Note that criteria are often established in advance without examining the alternatives. For example, what foods one should eat depends on body needs and tolerance and only after that is determined do we look for foods to satisfy these criteria. 
Second, the AHP derives relative ratio scales to empower the mind to deal with the real world in an understood, measured and proportioned way. It is a model that links the mind directly to experience. It gives answers that do not need intervention or further interpretation within normative principles imposed on peoples' thinking.

The use of ratio scales in the AHP to link, interpret and measure real happenings as they impact our value systems has a power of its own. The AHP departs from other ways of creating numbers to deal with the physical world in that when using it people are helped to decide on the meaning of data based on their own value system. Essentially the AHP is a unifying science that relates to our intrinsic values through ratio scale measurement, qualitative factors and numbers of all sorts, including hard measurements on ratio scales.

The AHP deals with structuring and measurement. Structuring involves five steps: 1) Identification; 2) Classification; 3) Clustering; 4) Ordinal ranking and stratification of levels under the goal; 5) Cardinal ordering of the elements in the levels. Measurement involves six steps: 1) Scales: absolute for comparisons, ratio for relative values; 2) Comparisons; 3) Judgment: verbal and numerical; 4) Priorities; 5) Consistency; 6) Synthesis.

There are three aspects of the AHP we will learn about in this meeting. The first is geographic: who, how and where is the AHP being used? The second is academic: how do we get more people in the academic community involved in teaching their students about the AHP, particularly in business and engineering schools? We also want social and political science students to know about our theory of measurement and how it can help.them with their research. Third and more generally, how do we get more scientists and mathematicians to examine relative measurement and its use in relating data to value systems, although these relations between data and values currentiy exist in the guise of an appropriately rationalized objective science.

Because some of us have felt that the AHP has broader implications than a specialized technique might, we have worked nonstop to simplify it and bring it to the attention of people in all walks of life. Occasionally we have had difficulty in getting articles accepted in journals controlled by editors from the older school of utility theory who don't seem pleased with our arrival on the scene. Referees of articles have told us: this is too simple, it cannot be true. Look how my complex method is, how hard it is to use even under the supervision of experts. How can a thing that simple be credible? An editor of Econometrica returned one of my papers 10 years ago with the following kind of comment by a referee: This is an ingenious way of doing things; but if we allow it in, what would we tell our students about the elaborate methods we teach them? Two other referees recommended that the paper be published, but simply because of this attitude, the conservative editor rejected the paper. Conversely, it is now obvious for all to see that anyone who wants to publish a criticism of the AHP in a journal whose editors are indoctrinated in utility theory with a circle of like minded referees who know little about the AHP can do so with few problems. Publication in these journals does not signify that one has a meaningful new result in the AHP or even that the work makes sense. New ideas need to be assessed with a particularly thoughtful eye.

The use of statistical methods in the AHP must be adapted and simplified to deal with a large number of participants. We need to place greater emphasis in our future meetings on agreeing on some useful directions for group decision making and statistical data processing and working together to develop and elucidate them to make them more readily accessible. We already made a start in that direction by writing about the subject and through the elaborate instrument of Team Expert Choice.

The AHP has created a wide opportunity for people to do research on the relationship 
between the factors in a problem and the value system in which it is embedded. From the diversity of applications, it seems that to the political, psychological and social world, the AHP serves a role similar to the contribution of the calculus in the technological world. I believe that the widespread use of the AHP in modelling can be attributed to the use of ratio scales needed to create meaning out of measurement and the manner in which complexity is decomposed in terms of clusters and homogeneous elèments in clusters represented in a coherent hierarchy.

\section{Comments on Applications of the AHP}

It is difficult to classify all the areas of human activity. The Hierarchon is an extensive anthology of hierarchies in a large diversity of problem areas that I put together with Ernest Forman but have myself so far revised three times. This anthology will be constantly updated from edition to edition to include new typical hierarchies. Many applications of the AHP are made on site by organizations and individuals. They range from a NASA application of choosing a propulsion systems for a lunar lander to health and medical, environmental and social applications, to an IBM application in the design of computers, to setting priorities for recipients of heart transplants, to the South Africa foreign office dealing with the conflict in that country, to applications in business and economics, in technology, architecture, transport, accounting and on and on. Perhaps a good way to familiarize oneself with the diverse areas is to examine the references at the end of my new book. We should remember that many corporate applications which are communicated informally or known by a number of us through consulting do not get included in the references.

A general approach to decision making is to combine the outcome of three hierarchies, one for benefits, one for costs and one for risks by taking the ratio of the priorities of the benefits of the alternatives to those of their costs and risks. Some problems can be dealt with by using one such hierarchy, some with two depending on what factors and structures are considered decisive for that problem. Some companies have used the forward backward process to plan for their future. In a recent paper predicting the turn around date of the U.S. economy in 1992 and how strong the recovery would be, I and my coauthors used a holarchy. A full blown supermatrix approach has been used to predict over a five year horizon, which company will be dominant in producing the central processing unit of a PC. I and my colleague Luis Vargas used the continuous version of the AHP to study neural firing in a paper included as a chapter at the end of my fundamentals book. Ami Arbel and Luis Vargas generalized the idea of point comparison to an interval of comparisons also extended by Hamalainen and his associates in Finland.

There are at least three software programs on hierarchies and one on Feedback. You will have an opportunity to learn about some of them in this meeting.

The supermatrix and the continuous approaches of the AHP are finding increasing demand. In addition to chapter 8 on dependence and feedback in the Fundamentals book, I am working on a book with extensive applications of the feedback approach. There are examples of the same problem with and without feedback with different outcomes. I believe that the feedback approach gives better results because of its faithfulness to capture dependence that is all around us. We have a new software for feedback developed by my student David Hauser which I use in teaching. I believe that the element of dependence and feedback is extremely important to introduce into decision making because in the real world things are interdependent and effects become causes. Our decomposition of problems into hierarchies is often a simplification that may not be fully justified. More generally, we need to look in greater depth 
simplification that may not be fully justified. More generally, we need to look in greater depth at the continuous formulation and how the mind can be assisted to respond to a large number of stimuli along lines compatible with one's perceptions.

\section{Technical Observations}

Here are some observations on a diversity of interests and technical points in the AHP.

\section{The Meaning and Non Meaning of Hard Measurements}

We all know that paired comparisons are made on pairs of homogeneous elements by using the smaller element as the unit and estimating the larger one as a multiple of it. People say how can we avoid using direct measurement data to get meaningful results. But what do the results mean and how do we use them? We need to recognize that arithmetic differences in data do not signify a parallel difference in judgment strength. We need to answer the following kind of questions. In my system of values, can I distinguish between lifting 3 pounds and lifting six pounds, or between lifting 50 pounds and lifting 100 pounds? Scientifically, does it make any difference for me to be able to make such a distinction? Does the numerical ratio or difference have the same degree of importance to me? Does greater difference have proportionately more importance, a lot more, less, or no difference to me? It is clear that the importance we assign to things does not literally parallel their measurement. How do we include such information in the AHP? We can use the ratios of the data directly as one does with judgments, or we can interpret the data with judgments for paired comparisons, and finally we can use ranges of the measurements among which we can make important distinctions to predict likely outcome in dollars or in months or the like. This applies equally to frequency of occurrence as probability. There are many examples of AHP applications in which the use of hard data is included in the analysis. The important observation is that we not only need the AHP to evaluate our own priorities of what we observe with our senses and through measurement, but we also need it to deal with intangibles for which no meaningful measurement exists. Converting everything to dollars is a poor way of dealing with complex problems. People's happiness, longevity, abilities, the art they produce, their love and hate are all reduced to dollars, a very deplorable practice. Setting priorities in terms of ratio scales is the AHP answer. Each problem is dealt with on the merit of its own objectives without resorting to some unit of measurement that is here today, and only in some cultures and ways of thinking, but gone tomorrow. In addition, the criterion of rationality that requires one to take more when offered more of that unit is untenable as one reaches saturation followed by reversal in the attractiveness of increasing values. A good theory should transcend transient interpretations.

Despite their apparent precision, hard data convey measurement on a scale which anyone can remember and repeat from memory but not necessarily understand without previous experience. Still the memory is useful for communicating numerical information.

A temperature of $30^{\circ}$ Fahrenheit or $-2^{\circ}$ Celsius means different things to different people. To an Eskimo this would feel warm while to a Kenyan who may never have experienced it, it is extremely cold and indistinguishable from $-30^{\circ} \mathrm{F}$. And, if as the convention is in science, if one attempts to convey the idea in terms of freezing of substances, like water, again, unless one has experienced frozen water, the significance of the example may be lost.

Finally, a number does not always convey the full idea of what one has in mind. To build a house one needs a certain area of a few hundred square feet - say 400 . Imagine what kind of house one would build on a 400 sq. ft. area that is one foot wide by 400 feet long. It 
is no use simply saying $400 \mathrm{sq}$. ft. Even when we describe $400 \mathrm{sq}$. ft. as 20 by 20 feet, we need much previous experience and understanding about the layout of a house. However, a 20 $\times 20$ square may not be an ideal lot for building a house on the side of a mountain. Numbers alone are not enough.

\section{Hard Measurements, Hierarchies and The Supermatrix}

In situations where hard measurements exist for the criteria and all of them belong to the same ratio scale, one cannot take each set of data, normalize it to relative form and weight it by criteria priorities. In this situation the criteria weights depend on the measurements of the alternatives under each criterion and the axiom of hierarchic independence in the AHP is violated. By ignoring the axiom, people have constructed examples erroneously claiming that 'hierarchic composition is wrong. There are in fact two equivalent ways to proceed. The first is to appropriately group those criteria for which alternatives are measured on the same ratio scale into a single unifying criterion and then determine weights for the criteria as usual (see Fundamentals of Decision Making). The second uses the supermatrix to compare those measurable criteria with respect to each alternative. The supermatrix would then also automatically deal with other criteria that may or may not be dependent on the alternatives for their values. We note that the importance of a criterion does not derive from the range of values of the alternatives under that criterion. This belief shared by utility practitioners stems from the use of interval scales which makes it difficult to derive criteria weights when one does not have units to compute marginal rates of substitution. In multiattribute utility theory the criteria weights do not reflect their importance. They are labelled as scaling constants. In utility theory, it is imperative that criteria have scales of absolute measurement with which to do marginal substitutions which are tradeoffs between units.

\section{Stability of the Eigenvector Requires a Small Number of Homogeneous Elements}

People have often said that the reason for comparing $7^{\circ} \pm 2$ elements in $\mathrm{AHP}$ is because I got the idea from psychologist George Miller. But that is not the case. I found early on that when the number of elements is small, near consistency the eigenvector is stable. It is on page 192 of my first AHP book and I decided to include it here again to emphasize its importance. After I wrote that book I learned about Miller's observation from my colleagues in the social sciences at the Wharton School and I began citing it in an effort to make AHP more accessible and appealing to social scientists.

The question often arises, how sensitive the priorities given by the eigenvector components are to slight changes in the judgment values. Clearly, it is desirable that the priorities do not fluctuate widely with small changes in judgment. There are essentially three ways to test this sensitivity: (1) by finding a mathematical estimate of the fluctuation; (2) by deriving answers based on a large number of computer runs appropriately designed to test the sensitivity; (3) by a combination of the two, particularly when it is not possible to carry out the full demonstration analytically.

In the case of consistency, $\lambda_{\max }$ is equal to the trace of the matrix which consists of unit entries. In this case one would expect the eigenvector corresponding to the perturbed matrix to undergo an overall change by an amount inversely proportional to the size of the matrix.

In general, the eigenvalues of a matrix lie between its largest and smallest row sums. Changing the value of an entry in the matrix changes the corresponding row sum and has a tendency to change $\lambda_{\max }$ by an equal amount. However, since a change in the eigenvector should 
also be influenced by the size of the matrix, we expect that the larger the matrix, the smaller the change in each component.

We begin the analytical treatment of the question by considering a matrix $A$ with the characteristic equation

$$
\operatorname{det}(A-\lambda I)=\lambda^{n}+a_{1} \lambda^{n-1}+\cdots+a_{n}=0
$$

Following standard procedures, let $A+\varepsilon B$ be the matrix obtained by introducing a small perturbation in $A$. The corresponding characteristic equation is

$$
\operatorname{det}(A+\varepsilon B-\lambda I)=\lambda^{n}+a_{1}(\varepsilon) \lambda^{n-1}+\cdots+a_{n}(\varepsilon)=0
$$

where $a_{k}(\varepsilon)$ is a polynomial in $\varepsilon$ of degree $(n-k)$, such that $a_{k}(\varepsilon) \rightarrow a_{k}$ as $\varepsilon \rightarrow 0$.

Let $\lambda_{1}$ be the maximum simple eigenvalue corresponding to the characteristic equation of $A$. It is known in matrix theory that for small $\varepsilon$, there exists an eigenvalue of $A+\varepsilon B$ which can be expressed as the sum of a convergent power series, i.e.,

$$
\lambda_{1}(\varepsilon)=\lambda_{1}+k_{1} \varepsilon+k_{2} \varepsilon^{2}+\cdots
$$

Let $w_{1}$ denote the eigenvector of $A$ corresponding to $\lambda_{1}$ and let $w_{1}(\varepsilon)$ be the eigenvector of $A+\varepsilon B$ corresponding to $\lambda_{1}(\varepsilon)$. The elements of $w_{1}(\varepsilon)$ are polynomials in $\lambda_{1}(\varepsilon)$ and $\varepsilon$, and, since the power series for $\lambda_{1}(\varepsilon)$ is convergent for small $\varepsilon$, each element of $w_{1}(\varepsilon)$ can be represented as a convergent power series in $\varepsilon$. We may write

$$
w_{1}(\varepsilon)=w_{1}+\varepsilon z_{1}+\varepsilon^{2} z_{2}+\cdots
$$

If the matrix $A$ has linear elementary divisors, then there exist complete sets of right.and left eigenvectors $w_{l}, w_{2}, \ldots, w_{n}$ and $v_{l}, v_{2}, \ldots, v_{n}$, respectively, such that

$$
v_{i}^{T} w_{j}=0 \quad i \neq j
$$

Note that $w_{j}$ and $v_{j}$ are the $j$ th eigenvectors (right and left), and not the $j$ th components of the vectors.

The vectors $z_{i}$ can be expressed in terms of the $w_{j}$ as

$$
z_{i}=\sum_{j=1}^{n} s_{i j} w_{j}
$$

which, when substituted in the formula for $w_{1}(\varepsilon)$, gives

$$
w_{1}(\varepsilon)=w_{1}+\sum_{i=2}^{n} \sum_{j=1}^{n} t_{i j} \varepsilon^{j} w_{i}
$$

where the $t_{i j}$ are obtained by dividing the $s_{i j}$ by the coefficient of $w_{l}$.

The first order perturbations of the eigenvalues are given by the coefficient $k_{l}$ of $\lambda_{l}(\varepsilon)$. eigenvectors.

We now derive the expression for the first order perturbations of the corresponding

Normalizing the vectors $w_{j}$ and $v_{j}$ by using the euclidean metric we have

We know that

$$
\left|v_{j}^{T}\right|\left|w_{j}\right|=1
$$

$$
(A+\varepsilon B) w_{1}(\varepsilon)=\lambda_{1}(\varepsilon) w_{1}(\varepsilon)
$$
we have

If we substitute the expressions for $\lambda_{1}(\varepsilon)$ and $w_{1}(\varepsilon)$ obtained above and use $A w_{l}=\lambda_{l} w_{l}$, 


$$
\sum_{j=2}^{n}\left(\lambda_{j}-\lambda_{1}\right) t_{j l} w_{j}+B w_{1}=k_{1} w_{1}
$$

Multiplying across by $v_{1}^{T}$ and simplifying, we obtain

$$
k_{1}=v_{1}^{T} B w_{1} / \nu_{1}^{T} w_{1} \quad \text { for } j=1
$$

and

$$
t_{j l}=\left(v_{j}^{T} B w_{1} /\left(\lambda_{1}-\lambda_{j}\right) v_{j}^{T} w_{1}\right) \quad \text { for } j \neq 1
$$

where, as noted above, $k_{l}$ is the first order perturbation of $\lambda_{l}$ and

$$
\left|k_{1}\right|=\left(v_{1}^{T} B w_{1} / \nu_{1}^{T} w_{1}\right) \leq[B] / v_{1}^{T} w_{1}
$$

where $[\mathrm{B}]$ is the sum of the elements of $\mathrm{B}$.

Thus for sufficiently small $\epsilon$ the sensitivity of $\lambda_{1}$ depends primarily on $v_{1}^{T} w_{1} \cdot v_{1}^{T} w_{1}$ might be arbitrarily small. We show that is not the case.

The first order perturbation of $w_{l}$ is given by

$$
\begin{aligned}
\Delta w_{1} & =\varepsilon \sum_{j=2}^{n} t_{j l} w_{j} \\
& =\varepsilon \sum_{j=2}^{n}\left(v_{j}^{T} B w_{1} /\left(\lambda_{1}-\lambda_{j}\right) v_{j}^{T} w_{j}\right) w_{j} \\
& =\sum_{j=2}^{n}\left(v_{j}^{T}(\Delta A) w_{1} /\left(\lambda_{1}-\lambda_{j}\right) v_{j}^{T} w_{j}\right) w_{j} \text { where } \Delta A \equiv \varepsilon B
\end{aligned}
$$

The eigenvector $w_{l}$ will be very sensitive to perturbations in $A$ if $\lambda_{l}$ is close to any of the other eigenvalues. When $\lambda_{1}$ is well separated from the other eigenvalues and none of the $v_{i}^{T} w_{i}$ is small, the eigenvector $w_{l}$ corresponding to the eigenvalue $\lambda_{2}$ will be comparatively insensitive to perturbations in $A$. This is the case, for example, with skew-symmetric matrices $\left(a_{j i}=-a_{i j}\right)$.

The $v_{i}^{T} w_{i}$ are interdependent in a way which precludes the possibility that just one $1 / \nu_{i}^{T} w_{i} i=1,2, \ldots, n$ is large. Thus if one of them is arbitrarily large, they are all arbitrarily large.

However, we want them to be small, i.e., near unity. To see this let

$$
w_{i}=\sum_{j} c_{i j} v_{j} \text { and } v_{j}=\sum_{j} d_{i j} w_{j}
$$

where $\left|w_{i}\right|=\left|v_{i}\right|=1, i=1,2, \ldots, n$. It is easy to verify by substitution that

and

$$
c_{i j}=w_{j}^{T} w_{i} / v_{j}^{T} w_{j}
$$

Then

$$
d_{i j}=v_{j}^{T} v_{i} / v_{j}^{T} w_{j}
$$

$$
\begin{aligned}
v_{i}^{T} w_{i} & =\sum_{j} d_{i j} w_{j}^{T} \sum_{j} c_{i j} v_{j} \\
& =\sum_{j}\left(w_{j}^{T} w_{i}\right)\left(v_{j}^{T} v_{i}\right) / v_{j}^{T} w_{j}
\end{aligned}
$$

For $i=j$,

and

$$
w_{i}^{T} w_{i}=v_{i}^{T} v_{i}=1
$$




$$
v_{i}^{T} w_{i}=\left(v_{i}^{T} w_{i}\right)^{-1}+\sum_{j \neq i}\left(w_{j}^{T} w_{i}\right)\left(v_{j}^{T} v_{i}\right)\left(v_{j}^{T} w_{j}\right)^{-1}
$$

Since

we have

$$
w_{j}^{T} w_{i}=\cos \theta_{i j} \quad \text { and } \quad v_{j}^{T} v_{i}=\cos \phi_{i j}
$$

$$
\begin{aligned}
\left|\left(v_{i}^{T} w_{i}\right)^{-1}\right| & \left.\leq \mid v_{i}^{T} w_{i}\right)\left|+\sum_{j \neq i}\right|\left(v_{j}^{T} w_{j}\right)^{-1} \mid \\
& \leq 1+\sum_{j \neq 1}\left|\left(v_{j}^{T} w_{j}\right)^{-1}\right|
\end{aligned}
$$

which must be true for all $i=1,2, \ldots, n$. This proves that all the $v_{i}^{T} w_{i}$ must be of the same order.

We now show that for consistent matrices $\left(v_{1}^{T} w_{1}\right)^{-1}$ cannot be arbitrarily large. We have in the case of consistency

Therefore

$$
\begin{aligned}
v_{1}^{T} & =\left(1 / w_{13}, \ldots, 1 / w_{1 n}\right) / \sum_{i=1}^{n} 1 / w_{1 i} \\
w_{1}^{T} & =\left(w_{11}, \ldots, w_{1 n}\right)
\end{aligned}
$$

$$
\begin{aligned}
\left(v_{1}^{T} w_{1}\right)^{-1} & =\left[\left(1 / w_{11}, \ldots, 1 / w_{1 n}\right)\left(w_{11}, \ldots, w_{1 n}\right)^{T} / \sum_{i=1}^{n} 1 / w_{1 i}\right]^{-1} \\
& =\left[n / \sum_{i=1}^{n} 1 / w_{1 i}\right]^{-1}>n
\end{aligned}
$$

since $n / \sum_{i=1}^{n} 1 / w_{1 i}<\sum_{i=1}^{n} w_{1 i} / n$

Now $\left(v_{1}^{T} w_{1}\right)^{-1}$ is minimized when all $w_{l i}$ are equal since $\sum_{i=1}^{n} w_{1 i}=1$. In practice, to $\operatorname{keep}\left(v_{1}^{T} w_{1}\right)^{-1}$ near its minimum we must deal with relatively comparable activities so that no single $w_{l i}$ is too small.

To improve consistency the number $n$ must not be too large. On the other hand, if we are to make full use of the available information and produce results which are valid in practice, $n$ should also not be too small. If, for example, we reject the values $v_{1}^{T} w_{1} \leq 0.1$, then we must have $n \leq 9$.

Under the assumption that the number of activities being compared is small and that they are relatively comparable, i.e., their weights differ by a multiple of their number, we can show that none of the components of $w_{l}$ is arbitrarily small and none of those of $v_{l}$ is arbitrarily small, and hence the scalar product of the two normalized vectors cannot be arbitrarily small.

With large inconsistency one cannot guarantee that none of the $w_{l i}$ is arbitrarily small. Thus, near-consistency is a sufficient condition for stability. Note also that we need to keep the number of elements relatively small, so that the values of all the $w_{l i}$ are of the same order.

\section{The Issue of Rank Preservation and Reversal}

The literature contains numerous examples of rank reversal. Those who developed the examples have not been able to justify them in terms of new criteria, or change in criteria 
weights. One may ask what can rank reversal possibly be attributed to. It has long been held that the method itself should not be responsible for allowing rank to change for then it would be regarded as arbitrary. I believe that is not correct. If a method is responsible for preserving yrank because people want it to, and if rank reversal does take place, then a method must also allow rank to reverse as needed. Rank reversal takes the process from the control and supervision of people and in part places the burden on the method itself to do it correctly. Thus. one must understand the process of rank preservation and reversal and create the appropriate methodology, to let each occur as necessary. When one adds alternatives, the character of the original set of alternatives is affected by the change in number or by the quality of a new alternative which now affects each alternative as a member of the new family. For example a student's learning ability is different depending on the number and quality of other students present. The criteria we use to compare alternatives are intrinsic attributes relating to each alternative; a structural property does not belong to an alternative and must be accounted for by the method of ranking itself. Hence the use of normalization in the AHP.

Because of the many published counterexamples, it is now agreed by everyone including utility theorists that rank reversal occurs in real life. These examples cannot be explained by the conventional arguments attributing rank reversal to criteria, their weights and to changes in judgment. There is no good theory proposal to explain rank reversal in utility theory. Always preserving rank can give the wrong answers. It is not difficult to see that rank reversal is due to other reasons that arise from the alternatives taken as a group; that one cannot rank an - alternative by looking at other alternatives without violating the independence assumption; that there are two kinds of dependence among the alternatives, one due to their function and one due to the structure of the problem; that structural effects must be captured by the method used and not by manipulation of criteria and judgments. Including uniqueness as a criterion involves the assumption of dependence of the alternatives. In addition, if the effect of copies occurs over 5th, 17th and 43rd times what sort of criteria would one use for that purpose? If a copy can cause rank reversal, then by small perturbation any alternative can cause rank reversal. Here is an example:

If a copy can convincingly cause rank reversal and a near copy also can, then a series of perturbations leading to some far off alternative also can give rise to rank reversal as the example below shows. It is easy to see this by considering alternatives $A_{j}$ rated with relative values on equally weighted criteria $C_{i}$. Here the alternative $A_{3.1}$ is a copy of $A_{3}$ and $A_{3.2}, A_{3.3}$, $A_{3,4}$ and $A_{3,5}$ are each a perturbation of the preceding one, producing changes in rank and in weight. Each column is normalized, weighted and the rows summed.

\begin{tabular}{|c|c|c|c|c|c|c|}
\hline \multirow[b]{2}{*}{$A_{1}$} & \multicolumn{4}{|c|}{ 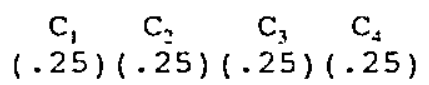 } & \multirow{2}{*}{$\frac{\text { Rank }}{3}$} & \multirow{2}{*}{$\begin{array}{l}\text { Weight } \\
.320\end{array}$} \\
\hline & 1 & 9 & 1 & 3 & & \\
\hline$A=$ & 9 & 1 & 9 & 1 & 2 & .336 \\
\hline \multirow[t]{2}{*}{$A_{3}$} & 8 & 1 & 4 & 5 & 1 & .344 \\
\hline & $c_{1}$ & $\mathrm{C}_{2}$ & $c_{3}$ & $\mathrm{C}_{4}$ & Rank & Weight \\
\hline \multirow{5}{*}{$\begin{array}{l}A_{1} \\
A_{2} \\
A_{3} \\
A_{3.1}\end{array}$} & 1 & 9 & 1 & 3 & 1 & .265 \\
\hline & 9 & $I$ & 9 & 1 & 2 & .250 \\
\hline & 8 & 1 & 4 & 5 & 3 & .243 \\
\hline & 8 & 1 & 4 & 5 & 3 & .243 \\
\hline & $c_{1}$ & $\mathrm{C}_{2}$ & $c_{3}$ & $\mathrm{C}_{4}$ & Rank & Weight \\
\hline
\end{tabular}




\begin{tabular}{|c|c|c|c|c|c|c|}
\hline \multirow{5}{*}{$\begin{array}{l}A_{1} \\
A_{2} \\
A_{3} \\
A_{3.2}\end{array}$} & \multirow{5}{*}{$\begin{array}{l}1 \\
9 \\
8 \\
7\end{array}$} & & \multirow[b]{2}{*}{1} & \multirow[b]{2}{*}{3} & \multicolumn{2}{|c|}{1} \\
\hline & & & & & 1 & .264 \\
\hline & & 1 & 9 & 1 & 2 & .247 \\
\hline & & 1 & 4 & 5 & 3 & .246 \\
\hline & & 1 & 5 & 5 & 4 & .243 \\
\hline \multirow{5}{*}{$\begin{array}{l}A_{1} \\
A_{2} \\
A_{3} \\
A_{3.3}\end{array}$} & $\mathrm{C}_{1}$ & $\mathrm{C}_{2}$ & $c_{3}$ & $\mathrm{C}_{4}$ & Rank & Weight \\
\hline & 1 & 9 & 1 & 3 & 1 & .264 \\
\hline & 9 & 1 & 9 & 1 & 3 & .245 \\
\hline & 8 & 1 & 4 & 5 & 4 & .243 \\
\hline & 6 & 1 & 6 & 5 & 2 & .248 \\
\hline \multirow{5}{*}{$\begin{array}{l}A_{1} \\
A_{2} \\
A_{3} \\
A_{3.4}\end{array}$} & $c_{1}$ & $\mathrm{C}_{2}$ & $\mathrm{C}_{3}$ & $\mathrm{C}_{4}$ & Rank & Weight \\
\hline & 1 & 9 & 1 & 3 & 1 & .264 \\
\hline & 9 & 1 & 9 & 1 & 4 & .244 \\
\hline & 8 & 1 & 4 & 5 & 3 & .245 \\
\hline & 5 & 1 & 7 & 5 & 2 & .248 \\
\hline \multirow{5}{*}{$\begin{array}{l}A_{1} \\
A_{2} \\
A_{3} \\
A_{3.5}\end{array}$} & $c_{1}$ & $\mathrm{C}_{2}$ & $c_{3}$ & $c_{4}$ & Rank & Weight \\
\hline & 1 & 9 & 1 & 3 & 1 & .264 \\
\hline & 9 & 1 & 9 & 1 & 4 & .243 \\
\hline & 8 & 1 & 4 & 5 & 2 & .246 \\
\hline & 4 & 1 & 8 & 5 & 2 & .246 \\
\hline
\end{tabular}

One of the papers written about the cause of rank reversals in utility theory concludes that reversals occur due to procedural invariance. In other words the same procedure is used without regard as to whether it should or should not happen. In fact there are situations in which it is not reasonable for rank reversal to take place and others with an identical abstract setting as the first where it is not reasonable to preserve rank.

Formerly, we used absolute measurement to preserve rank as a normative requirement and used relative measurement to allow rank to change. In the last two years we have extended relative measurement to handle both outcomes, each requiring a slightly different procedure. The ideal mode of the AHP preserves rank from irrelevant alternatives and the distributive mode allows rank to change. In this manner we have resolved the rank reversal issue in relative measurement.

\section{Bayes Theorem and Decision Analysis}

In the fall of 1993 and after I made two trips to Stanford University to compare notes on how the AHP works and how decision analysis with Bayes theorem works, Luis Vargas and I proved that the AHP implies Bayes Theorem. This result is included in chapter 8 of my book. We also gave an example which shows that assumptions of independence in Bayes Theorem when forced on problems with dependence can lead to wrong conclusions. A patient in a hospital in Youngstown, Ohio had his stomach removed by doctors who used decision analysis and Bayes Theorem on the assumption of independence of his symptoms. It was later realized that he could have been treated with medication.

\section{Multilinear Forms - The Nonlinearity of Hierarchic Composition}

The composite priorities of each alternative at the bottom level of a hierarchy may be represented as a multilinear form: 


$$
\sum_{i_{1} \cdots, i_{r}} x_{1}^{i_{1}} x_{2}^{i_{2}} \cdots x_{p}^{i_{p}}
$$

Consider a single term of this sum and for simplicity denote it by $x_{1}, x_{2}, \ldots, x_{p}$. We have

$$
x_{1} x_{2} \cdots x_{p}=e^{\log x_{1} x_{2} \cdots x_{r}}=\prod_{i=1}^{n} e^{\log x_{t}}=e^{\sum_{i=1}^{\infty} \log x_{1}} \rightarrow e^{\int \log x(\omega) d x}
$$

a product integral. Also

$$
x_{1} x_{2} \cdots x_{p}+y_{1} y_{2} \cdots y_{p}+\cdots+z_{1} z_{2} \cdots z_{p} \rightarrow \int_{\Lambda} e^{\int_{\lambda_{2}}^{\log u d u_{1}}} d \mu(\omega(\lambda))
$$

This is the same result as one obtains from the continuous formulation of hierarchic composition with eigenfunctions

$$
\begin{aligned}
& \int w_{n-1, n}\left(x_{n-1}, x_{n}\right) \int w_{n-2, n-1}\left(x_{n-2}, x_{n-1}\right) \cdots \int w_{1.2}\left(x_{1}, x_{2}\right) w_{1}\left(x_{1}\right) d x_{1} d x_{2} \cdots d x_{n} \\
& =\iint \cdots \int w_{n-1, n}\left(x_{n-1}, x_{n}\right) \cdots w_{1,2}\left(x_{1}, x_{2}\right) w_{1}\left(x_{1}\right) d x_{1} \cdots d x_{n} \\
& =\iint \cdots \int e^{\sum \log _{1-1,1}\left(x_{1,-1}, x_{1}\right)} d x_{1} \cdots d x_{n} \\
& \rightarrow \iint \cdots \int e^{\int \log \alpha_{i} d x_{i}} \cdot d x_{1} \cdots d x_{n}
\end{aligned}
$$

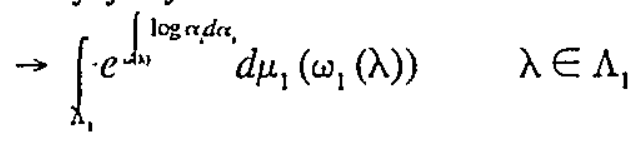

\section{Sensitivity and the Derivative}

We have used the integral operator in the AHP as a generalization of the basic eigenvalue

$\therefore$ structure. Thus we write

with the reciprocal property

$$
\int K(s, t) w(s) d s=\lambda_{\max } w(t)
$$

$$
K(s, t) K(t, s)=1
$$

With integration is associated the inverse operation of differentiation. We have not succeeded as yet to define the derivative in such a way as to link the AHP directly to the infinitesimal calculus. In its definition, the derivative relies on taking differences. But we want to always take ratios. Logarithms can be used for this purpose, but it is unclear what relation if any exists between the resulting definition of derivative to its usual definition in mathematical analysis. What we need is an analytical link between sensitivity analysis and differentiation as they relate to the multilinear forms of the AHP. It is likely that the best approach to the idea of derivative and rate of change would be obtained from Sobolev spaces where the eigenfunctions corresponding to neural firing belong. In that case one would be thinking of derivatives of Dirac type distributions.

\section{Closure}

In closing, I hope that we will all find this meeting enjoyable and have the opportunity to exchange ideas and points of view and learn from each other. Our subject is rooted in philosophy, the behavioral sciences, mathematics and in practice. It is hard for an individual to focus on the merit of an idea from one angle no matter how attractive the perspective may 
appear. Without the full interdisciplinary approach that perspective may be unacceptable. This caveat presents a broad challenge for us at this symposium and in our work in the future. Enjoy this meeting. I am including in the proceedings, following this introduction, a recent version of the theory and practice of the AHP for the non-technical person. 